\title{
Incidence, Predictors, and Success of Ventricular Tachycardia \\ Catheter Ablation in Arrhythmogenic Right Ventricular \\ Cardiomyopathy (From the Nordic ARVC Registry)
}

Morten K Christiansen, MD PhD ${ }^{\mathrm{a}}$, Kristina H Haugaa, MD PhD ${ }^{\mathrm{b}}$, Anneli Svensson, $\mathrm{MD}^{\mathrm{c}}$, Thomas Gilljam, MD PhD ${ }^{\mathrm{d}}$, Trine Madsen, MD PhD ${ }^{\mathrm{e}}$, Jim Hansen, MD PhD ${ }^{\mathrm{f}}$, Anders G Holst, MD PhD , Henning Bundgaard, MD DMSc ${ }^{\mathrm{g}}$, Thor Edvardsen, MD PhD ${ }^{\mathrm{b}}$, Jesper H Svendsen, MD PhD ${ }^{\mathrm{g}}$, Pyotr G Platonov, MD PhD ${ }^{\text {h }}$, Henrik K Jensen, MD DMSc ${ }^{\mathrm{a}}$

a Department of Cardiology, Aarhus University Hospital, Denmark \& Department of Clinical Medicine, Health, Aarhus University, Denmark

b Centre for Cardiological Innovation, Department of cardiology, Oslo University Hospital, Rikshospitalet, Norway and Institute for Clinical Medicine, University of Oslo, Oslo, Norway

c Department of Cardiology and Department of Medical and Health Sciences, Linköping University, Linköping, Sweden.

d Department of Cardiology, Sahlgrenska University Hospital, Gothenburg, Sweden

e Department of Cardiology, Aalborg University Hospital, Aalborg, Denmark

f Department of Cardiology, Herlev-Gentofte Hospital, University of Copenhagen, Copenhagen, Denmark

g Department of Cardiology, The Heart Centre, Rigshospitalet, Copenhagen, Denmark \& Department of Clinical Medicine, Faculty of Health and Medical Sciences, University of Copenhagen, Copenhagen, Denmark

h Department of Cardiology, Clinical Sciences, Lund University, Lund, Sweden

Short title: VT ablation in ARVC patients

Correspondence:

Morten Krogh Christiansen, MD PhD

Department of Cardiology, Aarhus University Hospital

Palle Juul-Jensens Boulevard 99, DK-8200 Aarhus, Denmark

Tel. +45 78452262 • Fax +45 78452260 • Email: morten.christiansen@ clin.au.dk 


\begin{abstract}
Catheter ablation may reduce ventricular tachycardia (VT) burden in arrhythmogenic right ventricular cardiomyopathy (ARVC) patients. However, little is known about factors predicting need for ablation. Therefore, we sought to investigate predictors and use of VT ablation and to evaluate the post-procedural outcome in ARVC patients. We studied 435 patients from the Nordic ARVC registry including 220 probands with definite ARVC according to the 2010 task force criteria and 215 mutation-carrying relatives identified through cascade screening. Patients were followed until first-time VT ablation, death, heart transplantation, or January 1st 2018. Additionally, patients undergoing VT ablation were further followed from the time of ablation for recurrent ventricular arrhythmias. The cumulative use of VT ablation was 4\% (95\%CI 3\%-6\%) and $11 \%$ (95\% CI 8\%-15\%) after 1 and 10 years. All procedures were performed in probands in whom cumulative use was $8 \%$ (95\%CI 5\%-12\%) and 20\% (95\%CI 15\%-26\%). In adjusted analyses among probands, only young age predicted ablation. In patients undergoing ablation, risk of recurrent arrhythmias was 59\% (95\%CI 44\%-71\%) and 74\% (95\%CI 59\%-84\%) 1 and 5 years after the procedure. Despite high recurrence rates, the burden of ventricular arrhythmias was reduced after ablation ( $\mathrm{p}=0.0042)$. Young age, use of several antiarrhythmic drugs and inducibility to VT after ablation were associated with an unfavorable outcome. In conclusion, twenty percent of ARVC probands developed a clinical indication for VT ablation within 10 years whereas mutationcarrying relatives were without such need. Although the burden of ventricular arrhythmias decreased after ablation, risk of recurrence was substantial.
\end{abstract}

\title{
Keywords
}

Arrhythmogenic Right Ventricular Dysplasia [MeSH]; Tachycardia, Ventricular [MeSH]; Ventricular Fibrillation [MeSH]; Arrhythmias, Cardiac [MeSH]; Catheter Ablation [MeSH] 


\section{Introduction}

Current guidelines recommend implantable cardioverter defibrillator (ICD) implantation as firstline therapy for the treatment of ventricular tachycardia (VT) in arrhythmogenic right ventricular cardiomyopathy (ARVC) patients at intermediate or high risk of future ventricular arrhythmias. ${ }^{1}$ Severely affected patients with incessant VT or frequent appropriate ICD interventions on maximally tolerated antiarrhythmic drugs (AAD) therapy may also be candidates for VT catheter ablation. ${ }^{1}$ Recently, a risk calculator to predict first-time episodes of ventricular arrhythmias in ARVC patients has been developed. ${ }^{2}$ However, there have been no studies reporting the risk of a need for VT ablation in ARVC patients. Consequently, it is unclear what characterizes the subgroup of patients at increased risk of developing a severe disease state requiring ablation. Moreover, several studies have reported varying results on the procedural efficacy. ${ }^{3-12}$ These studies have predominantly been conducted at large referral centers specialized in VT ablation and therefore the results may not reflect the general effect of VT ablation due to the selection and high volume in these centers. Therefore, the purpose of this study was to evaluate predictors and use of VT ablation and the post-procedural long-term outcome in a large multicenter cohort of patients with ARVC.

\section{Methods}

We conducted an observational cohort study using the Nordic ARVC Registry. The registry contains data on ARVC patients from eight institutions specialized in inherited cardiac diseases across Denmark, Norway, and Sweden (covering a population of approximately 14 million inhabitants). Patients with ARVC according to the 1994 Task Force Criteria (TFC) ${ }^{13}$ were retrospectively included in the registry when it was launched in June 2010, and since then patients fulfilling the $2010 \mathrm{TFC}^{14}$ have prospectively been included. For the present study we included patients (comprising both probands and relatives) in the registry fulfilling a diagnosis of definite ARVC according to the 2010 TFC. ${ }^{14}$ Genetic variants were classified according to the guidelines in force at the time of inclusion. In case a pathogenic mutation was found in the proband we also included mutation-carrying relatives identified through cascade screening following the mutation as far as possible in the respective families. These relatives were included irrespective of the TFC criteria, because we considered asymptomatic mutation-carriers to be in a subclinical disease state at risk of a need for future VT ablation. We followed all patients from the time of inclusion until the event of interest, death, heart transplantation, or January $1^{\text {st }} 2018$, whichever came first. In Denmark, registry studies do not require approval from an ethics committee, but approval was 
obtained from the Danish Data Protection Agency. The study complies with the Declaration of Helsinki.

Baseline clinical characteristics, data specific for the ARVC diagnosis as well as ventricular arrhythmic events and medical interventions are pre-specified registry variables. These data have prospectively been entered into the database upon clinical contacts according to the registry protocol since 2010. Historical information prior to the launch of the database has been entered based on information from the patients' medical records. Follow-up was performed regularly in all patients. In our centers, patients with definite ARVC are followed with symptomdriven examinations and seen at least annually. ${ }^{15}$ For the present study we extracted information on changes in AADs (including amiodarone, beta-blockers, sotalol, and other AADs) as well as data on ICD implantation and VT ablation, which are pre-specified reportable clinical events in the registry. The team of cardiac electrophysiologists at each site made the treatment indications including indications to perform VT ablation. VT ablations were performed using electro-anatomical mapping from 1998 and with the implementation of thermo-cool catheters from 2000 and onwards. VT ablation procedures were categorized as successful or unsuccessful depending on whether VT could be induced on repeated programmed ventricular stimulation immediately after the ablation procedure. Additional details regarding procedural access (i.e. endocardial, epicardial, or combined VT ablation procedure) and procedural complications were requested from each of the participating sites and collected from the medical records. We further retrieved events of sustained VT, ventricular fibrillation (VF), electrical storms defined as cases of three or more episodes of sustained VT/VF or appropriate ICD therapy per day, death (including causes of death), and heart transplantation. All episodes of VT/VF were documented by electrocardiogram- and/or ICD recordings.

Data are presented as mean \pm standard deviation (SD), median (interquartile range (IQR)) or number (proportion). Differences in baseline characteristics were evaluated using Wilcoxon's rank sum test, chi square test, or Fisher's exact as appropriate. We calculated the cumulative incidence of first-time VT ablation after 1, 5 and 10 years accounting for death and heart transplantation as competing risks. ${ }^{16}$ Cox proportional hazards regression was used to calculate unadjusted and adjusted hazard ratios (HRs) for potential predictors (age above median of 38 years, sex, proband status, sustained VT or aborted SCD at presentation, and mutation status) of first-time VT ablation. Probability weights were used to account for possible family clustering effects (except for analyses, which only included probands). The proportional hazards assumption was tested using log-log plots and found to be valid in all cases except for proband status since all events were observed among probands and none among relatives. Therefore, we restricted adjusted 
analyses to probands in which the remaining four predefined variables were included as covariates. All covariates were simultaneously added to the model. Since Cox regression was not a valid method to specifically address the difference in likelihood of VT ablation between probands and relatives we used a permutation test on the basis of 100.000 Monte Carlo simulations with matching based on age and sex to obtain a valid p-value. To evaluate the risk of recurrent arrhythmias as well as predictors of a favorable outcome after VT ablation we calculated the cumulative incidence of a composite endpoint of recurrent ventricular arrhythmias (appropriate ICD therapy, documented sustained VT or VF, electrical storm, aborted SCD, and SCD) after VT ablation at 1 and 5 years. Non- cardiac death, and heart transplantation were considered competing risks. Cox regression models with pre-specified subgroups (age above median at time of ablation, sex, number of AADs, endocardial- vs. epicardial or combined procedure, and immediate success after VT ablation) were used to evaluate potential predictors of a favorable outcome. Additionally, in a subgroup of patients with follow-up available 6 months before and after the ablation procedure, we investigated the registered burden of ventricular arrhythmias (appropriate ICD therapy, documented sustained VT or $\mathrm{VF}$, electrical storm, and aborted SCD). Wilcoxon's rank sum test was used to compare the number of registered events before and after the ablation procedure as well as the obtained reduction in the number of events between patients undergoing a successful and unsuccessful procedure, respectively. All analyses were performed using Stata version 13.1 (StataCorp, 4905 Lakeway Dr., College Station, TX, USA). Two-sided p-values $<0.05$ were considered statistically significant.

\section{Results}

We included 435 patients in the study. Baseline characteristics are shown in Table 1. Mean age was 38 years and 255 (59\%) were males. Relatives accounted for 215 (49\%) of the population, of which $91(42 \%)$ had definite ARVC. Baseline characteristics according to proband status are displayed in Supplementary Table 1 .

Over the time course of the study, a total of $90 \mathrm{VT}$ ablation procedures were registered in 49 patients. Median follow-up time from inclusion was 7.9 years (IQR 4.9-12.5 years). The cumulative incidence of VT ablation was 11\% (95\%CI 8\%-14\%) after 10 years (Table 2 and Figure 1). However, all ablation procedures were performed in probands. Comparing the use of VT ablation among pairs of probands and their corresponding relatives matched on age and sex reduced the number of patients for analysis to 80 , in which the likelihood of VT ablation was significantly higher in probands $(\mathrm{p}=0.0021)$. In univariate Cox regression models, male sex and ventricular arrhythmia at presentation were also significant predictors of the clinically considered need of VT 
ablation. However, these associations diminished in the adjusted analyses restricted to probands, where only age at inclusion below the median remained significantly associated with an increased clinically considered need of VT ablation.

Information on the VT ablation procedure as well as patient characteristics of the 49 patients undergoing first-time VT ablation is shown in Table 3. The use of VT ablation among ARVC patients increased steadily over the years with the first registered procedure performed in 1989 and $25(51 \%)$ of first-time procedures performed after January $1^{\text {st }} 2010$. The number of procedures performed per year is displayed in Supplementary Figure 1. Median follow-up time from VT ablation to the first event of interest, death or censoring was 0.8 years (IQR 0.2-3.9 years). The cumulative risk was high in all pre-specified subgroups (Figures 2 and Table 4), but younger age at the time of ablation and use of antiarrhythmic drugs were associated with a higher HR of ventricular arrhythmias. In adjusted analyses, these factors as well as inducibility to VT immediately after ablation were independently associated with an unfavorable outcome.

In 15 patients the first-time VT ablation procedure was performed within the first 6 months of follow-up after diagnosis, whereas one patient underwent heart transplantation less than 3 months after VT ablation. The remaining 33 patients were followed both 6 months before and 6 months after the ablation procedure. Among these patients the median number of registered episodes with ventricular arrhythmias was 2 (IQR 1-3, range 0-9) during the 6 months period prior to the first successful VT ablation compared with 0 (IQR 0-1, range 0-7) during the first 6 months period after ablation ( $\mathrm{p}=0.0042$ ). There was no change in the median number of antiarrhythmic drugs received at time of ablation compared with 6 months after ablation (median difference: 0 , IQR 0-0, range -1-1, p=1.00). A successful VT ablation was associated with a significant reduction in the number of ventricular arrhythmic episodes (Before: 2 (IQR 1-3, range 0-9) After: 0 (IQR 0-1, range $0-2$ ), $\mathrm{p}=0.0003$ ); a reduction not seen after unsuccessful procedures (Before: 1 (IQR 0-3, range 0-5) After: 0 (IQR 0-2, range 0-7), p=0.63) (Figure 3). However, comparing the reduction in the number of ventricular arrhythmic episodes between successful and unsuccessful procedures was not statistically significant $(\mathrm{p}=0.11)$.

In sensitivity analyses we further evaluated the effect of VT ablations over time as well as differences between participating centers on risk of recurrent ventricular arrhythmias (Supplementary Material). We found no statistically significant effects of time of ablation or experience with VT ablation at the participating sites on ventricular arrhythmia recurrence.

Information on VT ablation complications was available in 43 patients. Six (14\%) patients experienced a complication, of which 3 patients developed pericardial effusion, 1 developed embolism to the left popliteal artery, 1 developed traumatic circumflex artery injury 
treated with percutaneous coronary intervention, and 1 patient had polymorphic incessant VT induced by stimulation leading to cancellation of the procedure.

\section{Discussion}

The present study is the first to describe the use of VT ablation in a large multi-center registry of patients with an ARVC diagnosis. Our study showed that $20 \%$ of ARVC probands had a clinically driven VT ablation performed within 10 years after presentation, whereas no mutation-carrying relatives underwent any procedures. After first-time VT ablation we observed a substantial risk of recurrent ventricular arrhythmias in all predefined subgroups, although the number of ventricular arrhythmic events decreased notably after ablation. This reduction in ventricular arrhythmic burden was particularly driven by findings in patients in whom the VT ablation procedure was categorized as successful.

For the first person in a family to seek medical attention and to be diagnosed with ARVC (i.e. the proband) the phenotype needs to be clinically manifest. In case a pathogenic mutation is found, subsequent cascade screening may lead to the identification of several mutationcarrying relatives, in whom the disease may or may not be clinically evident. Accordingly, probands with definite ARVC have been shown to carry an 8-fold higher risk of ventricular arrhythmias compared with mutation-carrying relatives. ${ }^{17}$ Although this finding may partly be explained by the preclinical disease state in which relatives are often diagnosed another study also found a higher risk of ventricular arrhythmias in probands compared with relatives even though both probands and relatives all had definite ARVC. ${ }^{18}$ The absence of VT ablation procedures among mutation-carrying relatives in our study support prior findings by indicating that treatment with antiarrhythmic medication and ICD therapy according to current guidelines may be sufficient to prevent clinically significant ventricular arrhythmias in these individuals. Although mutationcarrying relatives a priori have a major ARVC criterion given their mutation, it is worth noting that 2 out of 5 mutation-carrying relatives in our study fulfilled the 2010 TFC for definite ARVC at the time of diagnosis. Nevertheless, these patients did not undergo VT ablation, suggesting a less penetrant disease expression in affected relatives compared with the proband in the family. In this context it should be emphasized that the presence of a mutation in itself does not seem to predict ventricular arrhythmias in patients with definite ARVC. ${ }^{2}$ Accordingly, in the present study mutation status was not associated with need for VT ablation among probands with definite ARVC. Consequently, the identification of a disease-causing mutation in a family might be seen as a confirmation of monogenic ARVC rather than a marker of outcome. 
Prior studies of patients undergoing VT ablation have shown a large variability in reported outcomes. ${ }^{3-12}$ In general the most promising results have been demonstrated in from highvolume single-centers using an epicardial ablation strategy, which has been increasingly utilized throughout the last decade. ${ }^{4,5,7,10}$ These studies have reported VT-free survivals ranging from $91 \%$ after a median follow up of 11 months to $71 \%$ after 56 months. ${ }^{4,5,7,10}$ In the light of these studies it is rather disappointing that 3 out of 4 patients in our cohort experienced a recurrent ventricular arrhythmia within 5 years. One explanation may be the continuing evolution of mapping and ablation techniques over the time course of the study, and therefore, these results might not reflect success rates currently achieved. However, we did not find evidence of improved outcomes over time, despite ventricular arrhythmic burden after ablation seemed to be more markedly reduced in later years. Furthermore, we were not able to demonstrate superiority of an epicardial or combined ablation approach, although it should be emphasized that only $16 \%$ of procedures used an epicardial access, which may in part explain the disappointing efficacy. Consequently, our results may reflect the general outcome expected after VT ablation among unselected ARVC patients seen in the broad daily clinical practice.

The major strength of our study includes the long-term follow-up on a large multinational cohort of patients with ARVC. However, our study has several limitations. The study was based on registry data from a large registry on ARVC, which does not contain detailed data on indications for VT ablation, mapping techniques, ablation procedures, or VT morphologies. The different TFC criteria used prior to and after 2010 might have caused a slightly different selection of patients over time. Also, since data prior to the launch of the registry in 2010 were based on retrospectively collected data from medical files there is a chance that clinically insignificant VT episodes have been underreported. However, we found the risk of recurrent ventricular arrhythmias to be similarly high in both time periods. Some patients (in particular the very elderly) may have been considered ineligible for VT ablation. Although this is likely not the case for the vast majority of the population, there is a potential risk of an indication bias slightly influencing the estimates. The rate of procedures at each center was relatively low in light of the long-term follow-up and the number of participating centers. Consequently, it might be possible that a higher experience with VT ablation in ARVC patients at the participating centers would have improved outcome after ablation. Over the course of the study guidelines and treatment protocols have changed. Hence, the results reflect those averaged over time and may not reflect current regimens. In order for a patient to be candidate for VT ablation, a ventricular arrhythmia needs to be present before but not after the time of the procedure. Therefore, some of the reduction in the ventricular arrhythmic burden may potentially be explained by a natural fluctuation in the VT burden over time giving rise to an 
overestimation of the effect of VT ablation. In the multivariable cox regression models the number of predictor variables was relatively high compared to the number of events giving rise to a potential risk of over-fitting the models. To address this issue we performed additional sensitivity analyses including each covariate one at a time. These results proved to be very similar to the reported results, which were also supported by the relative similarities between crude and adjusted analyses.

In conclusion, probands diagnosed with ARVC carry a high risk of future need for VT catheter ablation whereas mutation-carrying relatives seem to be without such need. This may suggest a favorable prognosis among relatives in which standard care, comprising close monitoring and possibly ICD- and AAD therapy, may be sufficient to protect these individuals from recurrent ventricular arrhythmias. In probands undergoing VT ablation the post-procedural arrhythmic burden was reduced but risk of recurrent ventricular arrhythmias remained substantial.

\section{Disclosures}

AGH reports personal fees from Novo Nordisk, outside the submitted work. JHS reports grants, personal fees and other from Medtronic, grants and personal fees from Biotronik, personal fees from Boehringer Ingelheim and Astra Zeneca, grants from Gilead, outside the submitted work. HKJ reports grants from Novo Nordisk Foundation (NNF18OC0031258), personal fees from Abbott and BiosenseWebster, outside the submitted work. MKC, KHH, AS, TG, TM, JH, HB, TE, and PGP have nothing to disclose. 


\section{References}

1. Corrado D, Wichter T, Link MS, Hauer R, Marchlinski F, Anastasakis A, Bauce B, Basso C, Brunckhorst C, Tsatsopoulou A, Tandri H, Paul M, Schmied C, Pelliccia A, Duru F, Protonotarios N, Estes NAM, McKenna WJ, Thiene G, Marcus FI, Calkins H. Treatment of arrhythmogenic right ventricular cardiomyopathy/dysplasia: an international task force consensus statement. Eur Heart J 2015;36:3227-3237.

2. Cadrin-Tourigny J, Bosman LP, Nozza A, Wang W, Tadros R, Bhonsale A, Bourfiss M, Fortier A, Lie ØH, Saguner AM, Svensson A, Andorin A, Tichnell C, Murray B, ZEPPENFELD K, van den Berg MP, Asselbergs FW, Wilde AAM, Krahn AD, Talajic M, Rivard L, Chelko S, Zimmerman SL, Kamel IR, Crosson JE, Judge DP, Yap S-C, van der Heijden JF, Tandri H, Jongbloed JDH, Guertin M-C, van Tintelen JP, Platonov PG, Duru F, Haugaa KH, Khairy P, Hauer RNW, Calkins H, Riele te ASJM, James CA. A new prediction model for ventricular arrhythmias in arrhythmogenic right ventricular cardiomyopathy. Eur Heart J 2019;40:1850-1858.

3. Dalal D, Jain R, Tandri H, Dong J, Eid SM, Prakasa K, Tichnell C, James C, Abraham T, Russell SD, Sinha S, Judge DP, Bluemke DA, Marine JE, Calkins H. Long-Term Efficacy of Catheter Ablation of Ventricular Tachycardia in Patients With Arrhythmogenic Right Ventricular Dysplasia/Cardiomyopathy. J Am Coll Cardiol 2007;50:432-440.

4. Garcia FC, Bazan V, Zado ES, Ren J-F, Marchlinski FE. Epicardial substrate and outcome with epicardial ablation of ventricular tachycardia in arrhythmogenic right ventricular cardiomyopathy/dysplasia. Circulation 2009;120:366-375.

5. Berruezo A, Fernández-Armenta J, Mont L, Zeljko H, Andreu D, Herczku C, Boussy T, Tolosana JM, Arbelo E, Brugada J. Combined endocardial and epicardial catheter ablation in arrhythmogenic right ventricular dysplasia incorporating scar dechanneling technique. Circ Arrhythm Electrophysiol 2012;5:111-121.

6. Philips B, Madhavan S, James C, Tichnell C, Murray B, Dalal D, Bhonsale A, Nazarian S, Judge DP, Russell SD, Abraham T, Calkins H, Tandri H. Outcomes of catheter ablation of ventricular tachycardia in arrhythmogenic right ventricular dysplasia/cardiomyopathy. Circ Arrhythm Electrophysiol 2012;5:499-505.

7. Santangeli P, Zado ES, Supple GE, Haqqani HM, Garcia FC, Tschabrunn CM, Callans DJ, Lin D, Dixit S, Hutchinson MD, Riley MP, Marchlinski FE. Long-Term Outcome with Catheter 
Ablation of Ventricular Tachycardia in Patients with Arrhythmogenic Right Ventricular Cardiomyopathy. Circ Arrhythm Electrophysiol 2015;8:1413-1421.

8. Vaseghi M, Hu TY, Tung R, Vergara P, Frankel DS, Di Biase L, Tedrow UB, Gornbein JA, Yu R, Mathuria N, Nakahara S, Tzou WS, Sauer WH, Burkhardt JD, Tholakanahalli VN, Dickfeld TM, Weiss JP, Bunch TJ, Reddy M, Callans DJ, Lakkireddy DR, Natale A, Marchlinski FE, Stevenson WG, Bella Della P, Shivkumar K. Outcomes of Catheter Ablation of Ventricular Tachycardia Based on Etiology in Nonischemic Heart Disease: An International Ventricular Tachycardia Ablation Center Collaborative Study. JACC: Clinical Electrophysiol 2018;4:11411150.

9. Bai R, Biase LD, Shivkumar K, Mohanty P, Tung R, Santangeli P, Carlos LS, Vacca M, Verma A, Khaykin Y, Mohanty S, Burkhardt JD, Hongo R, Beheiry S, Russo AD, Casella M, Pelargonio G, Santarelli P, Sanchez J, Tondo C, Natale A. Ablation of ventricular arrhythmias in arrhythmogenic right ventricular dysplasia/cardiomyopathy: Arrhythmia-free survival after endoepicardial substrate based mapping and ablationablation of ventricular arrhythmias in arrhythmogenic right ventricular dysplasia/cardiomyopathy arrhythmia-free survival after endoepicardial substrate based mapping and ablation. Circ Arrhythm Electrophysiol 2011;4:478-485.

10. Philips B, Riele te ASJM, Sawant A, Kareddy V, James CA, Murray B, Tichnell C, Kassamali B, Nazarian S, Judge DP, Calkins H, Tandri H. Outcomes and ventricular tachycardia recurrence characteristics after epicardial ablation of ventricular tachycardia in arrhythmogenic right ventricular dysplasia/cardiomyopathy. Heart Rhythm 2015;12:716-725.

11. Mahida S, Venlet J, Saguner AM, Kumar S, Baldinger SH, AbdelWahab A, Tedrow UB, Castelletti S, Pantazis A, John RM, McKenna WJ, Lambiase PD, Duru F, Sapp JL, ZEPPENFELD K, Stevenson WG. Ablation compared with drug therapy for recurrent ventricular tachycardia in arrhythmogenic right ventricular cardiomyopathy: Results from a multicenter study. Heart Rhythm 2019;16:536-543.

12. Mathew S, Saguner AM, Schenker N, Kaiser L, Zhang P, Yashuiro Y, Lemes C, Fink T, Maurer T, Santoro F, Wohlmuth P, Reißmann B, Heeger CH, Tilz R, Wissner E, Rillig A, Metzner A, Kuck KH, Ouyang F. Catheter Ablation of Ventricular Tachycardia in Patients With Arrhythmogenic Right Ventricular Cardiomyopathy/Dysplasia: A Sequential Approach. J Am Heart Assoc 2019;8:e010365. 
13. McKenna WJ, Thiene G, Nava A, Fontaliran F, Blomstrom-Lundqvist C, Fontaine G, Camerini F. Diagnosis of arrhythmogenic right ventricular dysplasia/cardiomyopathy. Task Force of the Working Group Myocardial and Pericardial Disease of the European Society of Cardiology and of the Scientific Council on Cardiomyopathies of the International Society and Federation of Cardiology. Br Heart J 1994;71:215-218.

14. Marcus FI, McKenna WJ, Sherrill D, Basso C, Bauce B, Bluemke DA, Calkins H, Corrado D, Cox MGPJ, Daubert JP, Fontaine G, Gear K, Hauer R, Nava A, Picard MH, Protonotarios N, Saffitz JE, Yoerger Sanborn DM, Steinberg JS, Tandri H, Thiene G, Towbin JA, Tsatsopoulou A, Wichter T, Zareba W. Diagnosis of arrhythmogenic right ventricular cardiomyopathy/Dysplasia: Proposed modification of the task force criteria. Circulation 2010;121:1533-1541.

15. Haugaa KH, Bundgaard H, Edvardsen T, Eschen O, Gilljam T, Hansen J, Jensen HK, Platonov PG, Svensson A, Svendsen JH. Management of patients with Arrhythmogenic Right Ventricular Cardiomyopathy in the Nordic countries. Scand Cardiovasc J 2015;49:299-307.

16. Satagopan JM, Ben-Porat L, Berwick M, Robson M, Kutler D, Auerbach AD. A note on competing risks in survival data analysis. British Journal of Cancer 2004;91:1229-1235.

17. Bhonsale A, James CA, Tichnell C, Murray B, Madhavan S, Philips B, Russell SD, Abraham T, Tandri H, Judge DP, Calkins H. Risk stratification in arrhythmogenic right ventricular dysplasia/cardiomyopathy-associated desmosomal mutation carriers. Circ Arrhythm Electrophysiol 2013;6:569-578.

18. Mazzanti A, Ng K, Faragli A, Maragna R, Chiodaroli E, Orphanou N, Monteforte N, Memmi M, Gambelli P, Novelli V, Bloise R, Catalano O, Moro G, Tibollo V, Morini M, Bellazzi R, Napolitano C, Bagnardi V, Priori SG. Arrhythmogenic Right Ventricular Cardiomyopathy: Clinical Course and Predictors of Arrhythmic Risk. J Am Coll Cardiol 2016;68:2540-2550. 


\section{Figure legends}

Figure 1. Cumulative incidence of VT catheter ablation stratified by subgroups Cumulative incidence functions calculated with death and heart transplantation as competing risks. A: All patients. B-F: Stratified by subgroups.

Figure 2. Cumulative risk of recurrent ventricular arrhythmias after first-time VT catheter ablation

Cumulative risk of ventricular arrhythmias including ventricular tachycardia (VT), ventricular fibrillation (VF), electrical storm and sudden cardiac death. Non-sudden cardiac death, non-cardiac death, and heart transplantation were taken into account as competing risks. A: All patients. B-F: Stratified by subgroups. AAD = antiarrhythmic drug.

\section{Figure 3. Burden of ventricular arrhythmias before and after VT catheter ablation} Number of VT/VF in patients with $\geq 6$ months follow-up before (blue) and after (red) VT catheter ablation, stratified in successful (A) and unsuccessful (B) procedures. VT/VF include registered episodes of ventricular tachycardia (VT), ventricular fibrillation (VF), and electrical storm. 